\title{
PROSPECÇÃO TECNOLÓGICA SOBRE MÉTODOS PARA DETERMINAÇÃO DA OXIDAÇÃO DE ÓLEOS E BIOCOMBUSTÍVEIS COM ÊNFASE EM MÉTODOS ÓTICOS
}

\author{
Alessandra S. Tanajura ${ }^{1 *}$, Marilena Meira ${ }^{2}$, Erika M. de O. Ribeiro ${ }^{1}$, Humbervânia R. Gonçalves ${ }^{1}$, \\ Cristina M. Quintella ${ }^{1}$ \\ ${ }^{1}$ Instituto de Química, Universidade Federal da Bahia, Campus de Ondina, 40170-290 Salvador- \\ BA, Brasil (alessandratanajura@gmail.com) \\ ${ }^{2}$ Instituto Federal da Bahia, Campus Simões Filho. Av Pitanguinhas s/n Simões Filho - Bahia
}

\begin{abstract}
RESUMO
Um problema importante associado ao biodiesel é a sua baixa estabilidade oxidativa, a oxidação pode causar acidez e aumento da viscosidade do combustível. Com o aumento gradual do uso de biodiesel como combustível surge a necessidade do desenvolvimento de novos métodos analíticos para a determinação da estabilidade oxidativa. Este trabalho tem como objetivos principais a busca de anterioridade para determinação da estabilidade oxidativa de óleos e biocombustíveis através de métodos óticos, incluindo espectrofluorimetria, e apresentar uma visão geral do estado atual de P\&D de tecnologias nesta área de estudo. A metodologia de pesquisa inicial consistiu na associação da palavra-chave oxidat* com o código G01N21, que se refere a métodos óticos sendo encontradas 296 patentes. Acrescentando nesta busca a palavra-chave oil são encontradas apenas 8 patentes e nenhuma destas patentes trata de espectrofluorimetria associada à quimiometria para determinação da estabilidade oxidativa de óleos e biodieseis.
\end{abstract}

Palavras Chave: Estabilidade Oxidativa, óleos, biodiesel, métodos óticos.

\begin{abstract}
One disadvantage associated with biodiesel is its poor oxidation stability. This disadvantage makes fuel unsuitable for use in engines because the resulting oxidation products can damage the motors of vehicles. Considering the gradual increase in the use of biodiesel as fuel, development of new analytical methods for determination of oxidative stability is a current need. This work has as main objectives to provide a search of prior patents for determination of oxidative stability of oils and biofuels through optical methods including spectrofluorimetry, and provide an overview of the present status of $\mathrm{P} \& \mathrm{D}$ of technologies in this area of study. The research methodology consisted of the associated keyword oxidat* with the code G01N21, which refers to optical methods. Adding in this search the keyword oil* are found only 8 patents and none of these patents reports the use of spectrofluorimetry associated with multivariate data analysis to prediction of the oxidation stability.
\end{abstract}

Key words: oxidation stability, oil, biodiesel, optical methods.

Área tecnológica: Biocombustíveis

Cadernos de Prospecção - ISSN 1983-1358 (print) 2317-0026 (online), 2012. vol.5, n.3, p.141-151

D.O.I.: 10.9771/S.CPROSP.2012.005.014 


\section{INTRODUÇÃO}

O biodiesel é uma mistura de ésteres alquílicos de ácidos graxos, geralmente feito por transesterificação de óleos vegetais ou gorduras animais com álcoois de cadeia curta, como metanol e etanol. Um problema importante associado com o biodiesel é a sua baixa estabilidade oxidativa. A química de oxidação do biodiesel é a mesma que a dos óleos e gorduras, a partir do qual foram obtidos, porque a cadeia de ácidos graxos não é alterada durante o processo químico em que ácidos graxos são transesterificados em ésteres alquílicos. Muitos dos óleos de origem vegetal contêm ácidos graxos de cadeia insaturada, como os ácidos linoléico e linolênico, que são a causa da instabilidade de óleos e biodiesel.

Entre os fatores que afetam a velocidade de oxidação incluem-se a concentração de oxigênio, o número de insaturações na cadeia, a presença de antioxidantes, presença de metais, a temperatura e a luz. A estabilidade oxidativa de um óleo ou biodiesel é favorecida pelo maior teor de ácidos graxos saturados (QUINTELLA et al., 2009). Quanto maior o número de insaturações na cadeia do ácido graxo mais suscetível ele será à oxidação. Isomerização de cis para trans pode afetar a estabilidade oxidativa porque a dupla na configuração trans apesar de ser mais estável é mais susceptível à oxidação que a dupla em cis (BERTHIAUME, 2006).

Nos combustíveis, algumas propriedades químicas e físicas podem ser afetadas pela oxidação (MONYEM et al., 2001) causando acidez e aumento da viscosidade devido à formação de gomas insolúveis que podem obstruir os filtros (MONYEM et al., 2001b). Isso torna o combustível inadequado para uso em motores.

Os ácidos graxos saturados possuem uma reatividade muito baixa à oxidação. No entanto, óleos animais, ricos em ácidos graxos saturados são menos estáveis à oxidação que os óleos vegetais. Esta controvérsia é explicada devido à ausência de antioxidantes naturais nos óleos animais (SENDZIKIENE et al., 2005).

O principal método atualmente em uso para a determinação da estabilidade oxidativa é o método Rancimat que mede o período de indução (BERTHIAUME, 2006). Outros métodos conhecidos são a medição do índice de acidez, índice de peróxido, análise sensorial, determinação de dienos conjugados, valor de carbonila, análise de voláteis, etc.

Este trabalho tem como objetivos principais a busca de anterioridade para determinação da estabilidade oxidativa de óleos e biocombustíveis através de técnicas espectroscópicas, incluindo espectrofluorimetria, e apresentar uma visão geral do estado atual de desenvolvimento cientifico e tecnológico relacionados ao uso de métodos óticos para determinação da oxidação de amostras diversas incluindo óleos e biodiesel. Através do mapeamento das patentes são avaliadas as perspectivas do uso de métodos óticos incluindo espectrofluorimetria, para a determinação da estabilidade oxidativa de óleos considerando suas vantagens e desvantagens, bem como, sugestões de estudos e tecnologias a serem desenvolvidas. São mostrados os métodos de busca em bancos de patentes e de definição de focos, além de exemplos concretos de buscas compreendendo a classificação por códigos de patentes e por palavras-chave. 


\section{METODOLOGIA}

A metodologia de pesquisa objetivando mapear o uso de métodos óticos para a determinação da oxidação de amostras consistiu na associação da palavra-chave oxidat* com o código G01N21 que se refere a métodos óticos. A base de dados escolhida foi a European Patent Office (EPO) que é uma base mundial de acesso livre usualmente escolhida para prospecção. Foi também feita a associação das palavras-chaves oxidat* oil* com o código G01N21 e a associação das palavraschaves oxidat* biodie* também com o código G01N21 visando especificar o tipo de amostra, respectivamente para óleos e biodiesel. As patentes foram importadas para o programa de mineração de texto Vantage Point ${ }^{\circledR}$, e os dados foram tratados aplicando Thesaurus e lógica Fuzzi.

A Tabela 1 apresenta a pesquisa realizada no banco de patentes do escritório europeu de patentes, Espacenet ${ }^{\circledR}$.

Tabela 1: Planilha de definição de escopo da pesquisa de acordo com as palavras-chave e códigos da Classificação Internacional de Patentes (CIP).

\begin{tabular}{|c|c|c|c|c|c|c|c|}
\hline oxidat* & oil* & dies* & biodies* & propert* & chemistry* & G01N21 & Total \\
\hline $\mathrm{X}$ & & & & & & $\mathrm{X}$ & 296 \\
\hline & & & & $\mathrm{X}$ & $\mathrm{X}$ & & 4974 \\
\hline & $\mathrm{X}$ & & & $\mathrm{X}$ & $\mathrm{X}$ & & 222 \\
\hline & $\mathrm{X}$ & & & $\mathrm{X}$ & $\mathrm{X}$ & $\mathrm{X}$ & 0 \\
\hline & & $\mathrm{X}$ & & $\mathrm{X}$ & $\mathrm{X}$ & & 14 \\
\hline & & & $\mathrm{X}$ & $\mathrm{X}$ & $\mathrm{X}$ & & 1 \\
\hline & $\mathrm{X}$ & & & $\mathrm{X}$ & $\mathrm{X}$ & $\mathrm{X}$ & 11 \\
\hline & $\mathrm{X}$ & & & $\mathrm{X}$ & & $\mathrm{X}$ & 1079 \\
\hline $\mathrm{x}$ & $\mathrm{X}$ & & & & & & 39080 \\
\hline $\mathrm{x}$ & & & $\mathrm{x}$ & & & $\mathrm{x}$ & 0 \\
\hline $\mathrm{x}$ & $\mathrm{X}$ & & & & & $\mathrm{x}$ & 8 \\
\hline
\end{tabular}

Fonte: Autoria própria, 2012.

\section{RESULTADOS E DISCUSSÃO}

Foram encontradas 296 patentes na busca pela associação da palavra chave oxidat* com o código G01N21 que se refere a investigar ou analisar materiais através do uso de métodos óticos, isto é, usando luz ultra-violeta, infravermelho ou visível. Destas patentes estavam disponíveis 200, as quais foram enviadas para o software Vantage Point ${ }^{\circledR}$. Estes resultados mostraram que existem 
relativamente poucas patentes depositadas relacionadas ao uso de métodos óticos para a determinação do grau de oxidação de amostras.

A Figura 1 revela que existe um crescente aumento do número de patentes nos últimos anos. A falta de dados nos anos de 2010 e 2011 é devida ao fato de não se dispor dos dados completos em função do período de 18 meses de sigilo das patentes.

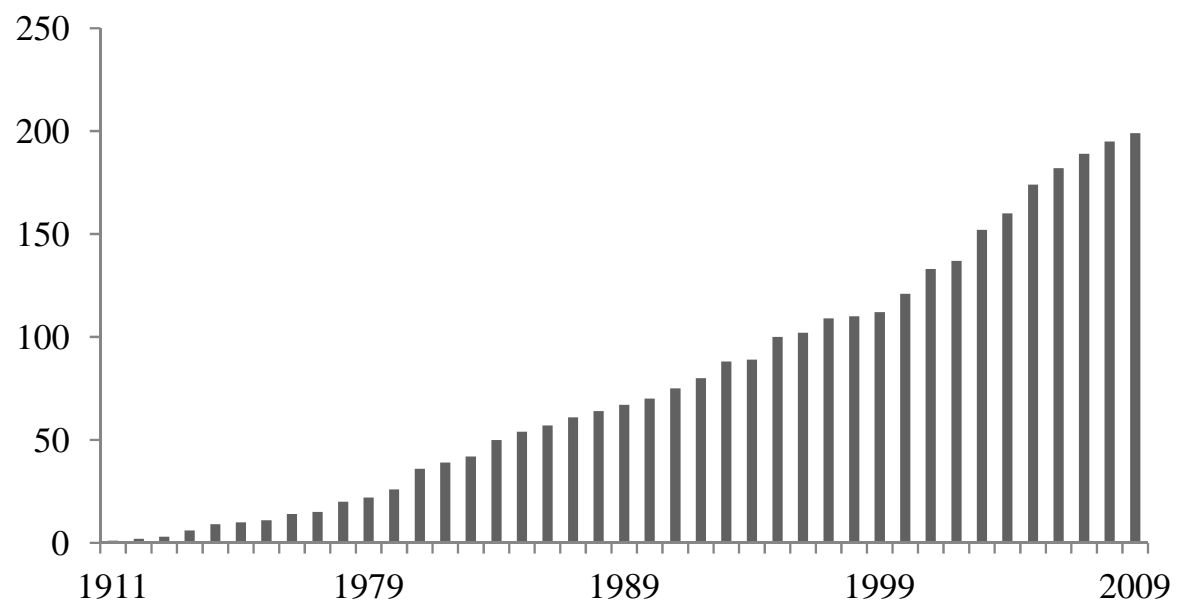

Figura 1: Distribuição das patentes depositadas no Espacenet por ano de depósito. Fonte: Autoria própria, 2012.

Os Estados Unidos, Japão e Alemanha são os grandes detentores da tecnologia relativa ao uso de métodos óticos para a determinação da oxidação de amostras. Esses resultados são apresentados na Figura 2. Pode-se destacar que o Brasil não aparece entre os maiores depositantes.

Através do uso do software Vantage Point analisou-se a possibilidade de existência de relacionamento entre os países que mais depositaram patentes, ou seja, se eles faziam algum tipo de parceria para produção das patentes encontradas. No entanto, como pode ser observado na Figura 3, cada país produziu suas patentes individualmente sem ocorrência de parcerias entre eles no que diz respeito ao tema estudado.

Entre os inventores que mais produziram, destaca-se Stamler Jonathan dos Estados Unidos com 6 patentes relativas a oxidação utilizando métodos óticos. Entre os depositantes destaca-se a empresa Fuji Photo Film uma das maiores produtoras de filmes fotográficos dos Estados Unidos com 6 patentes depositadas. A Fuji realiza pesquisas em diversas áreas de estudo, visando aumentar o campo de atuação.( http://www.fujifilm.com.br/)

Entre os depositantes (Figura 4), é possível também identificar o percentual de patentes que foram depositadas por cada um deles. Observa-se que as patentes estão distribuídas por vários, na havendo um depositante que seja claramente majoritário. 


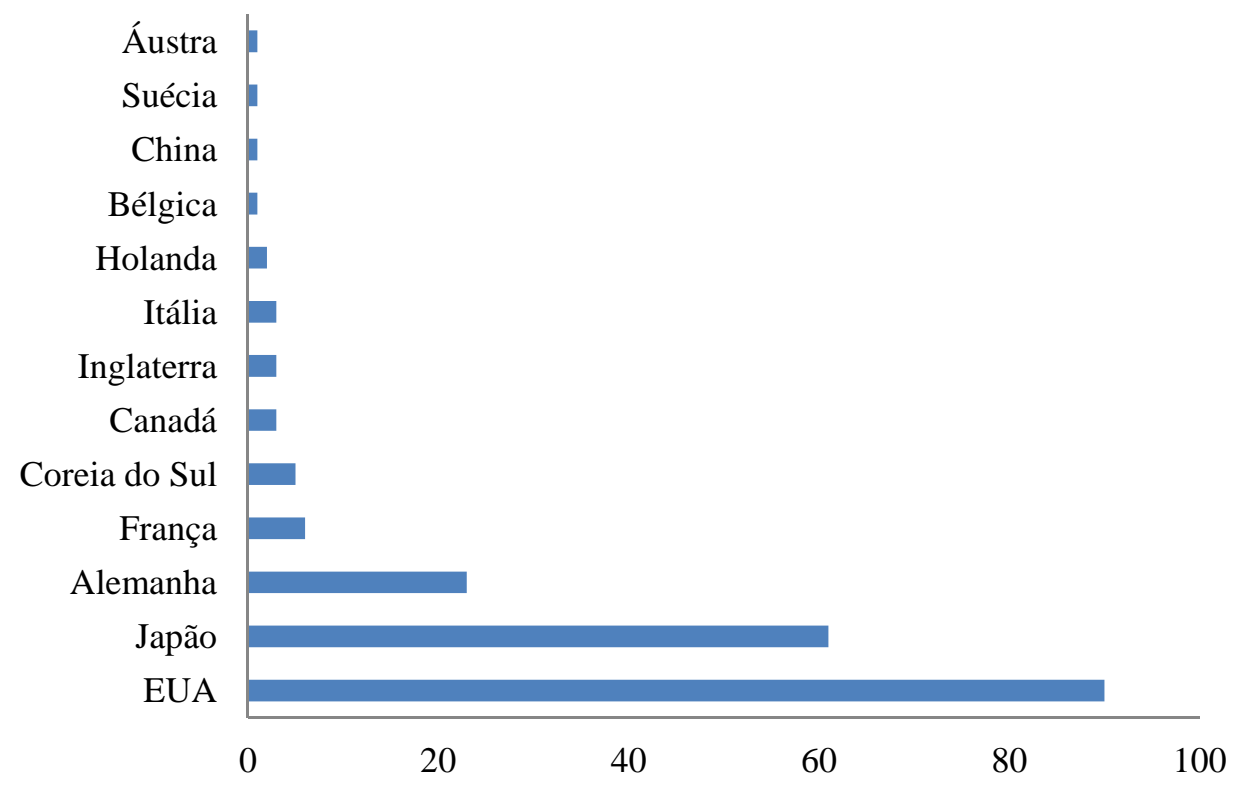

Figura 2:Distribuição das patentes depositadas no Espacenet por país de origem. Fonte: Autoria própria, 2012.

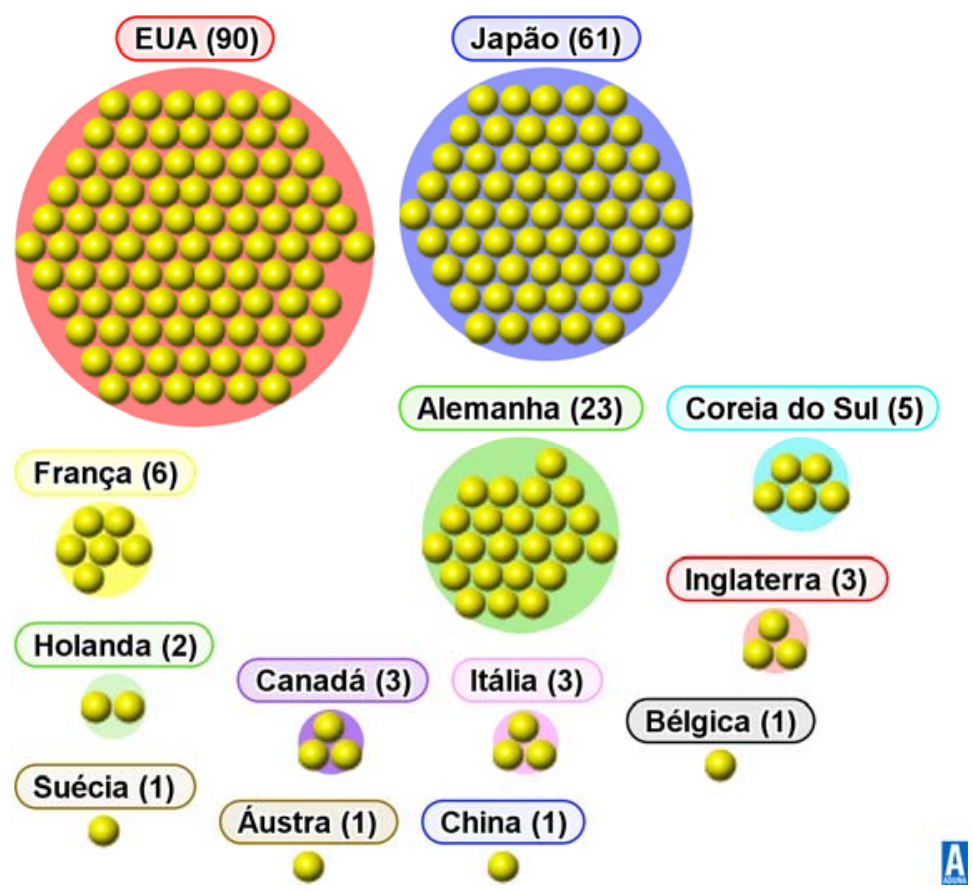

Figura 3 : Relação entre países depositantes de patentes relacionadas a métodos óticos e oxidação. Fonte: Autoria própria, 2012. 
Na Figura 7, os códigos de classificacao significam: G01N21/76 Quimiluminescência; Bioluminescência; G01N21/78 - produzindo uma alteração de cor; G01N31/00C - Investigação ou análise de materiais não biológicos pela utilização de métodos químicos especificados nos subgrupos (testando a eficiência dos procedimentos de esterilização sem a utilização de enzimas ou micro-organismos; G01N21/63C oticamente excitados - G01N21/33 utilizando luz ultravioleta; G01N21/76G - Quimiluminescência; Bioluminescência; G01N21/77 - pela observação do efeito sobre um indicador químico.

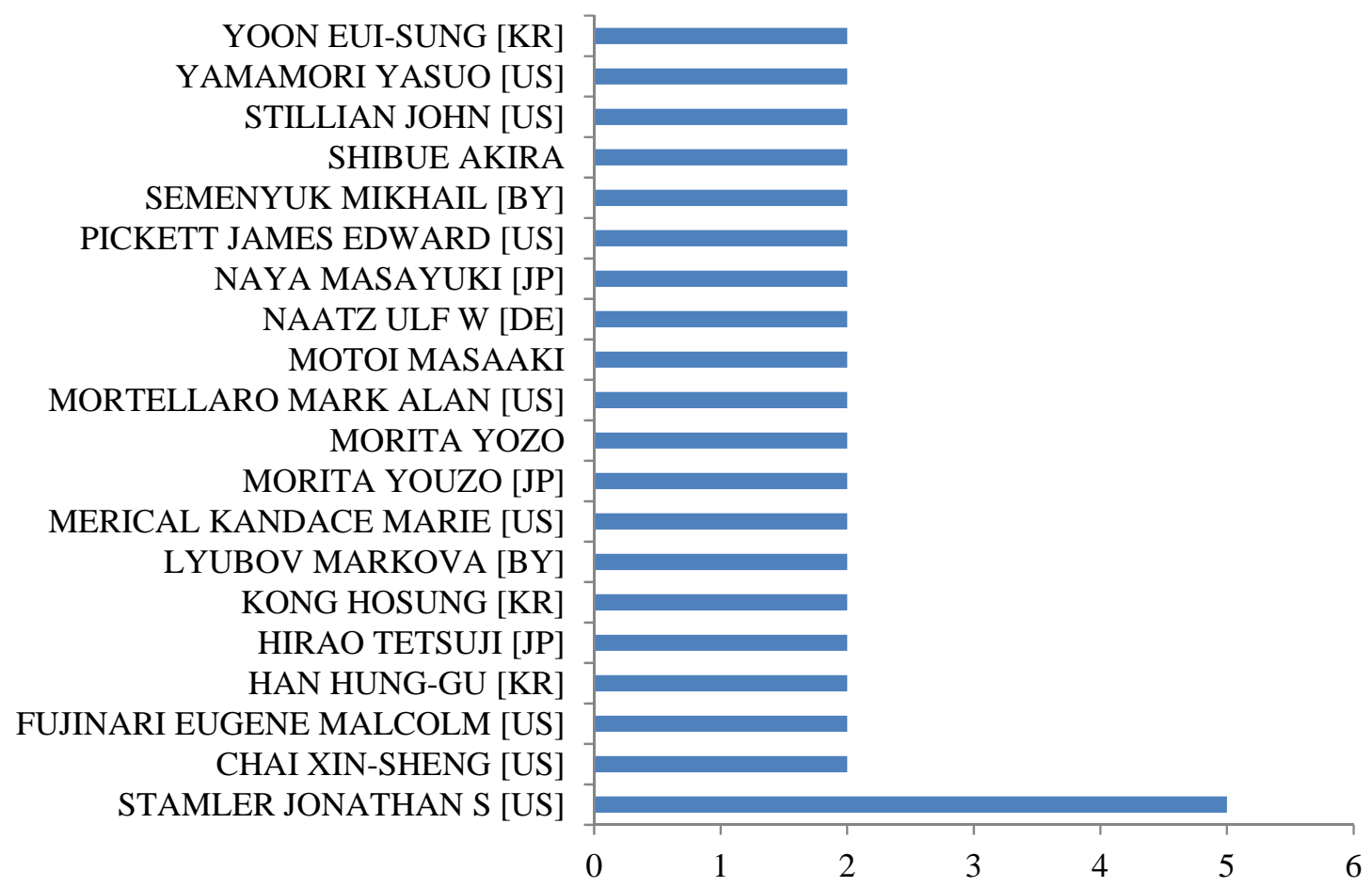

Figura 4: Distribuição das patentes depositadas no Espacenet por depositante. Fonte: Autoria própria, 2012.

As patentes são depositadas pela academia, por pessoas físicas, pelas corporações ou pelas instituições governamentais, conforme mostrado na Figura 5. Podemos observar que as empresas particulares detêm a maior fatia da tecnologia com $73 \%$ das patentes. As universidades e instituições governamentais possuem juntas 21\% das patentes e pessoas físicas apenas $6 \%$. A partir destes dados, podemos concluir que o interesse dos governos e universidades pela pesquisa de novos métodos para determinação da oxidação de amostras é muito baixo, quando comparado com as empresas. 


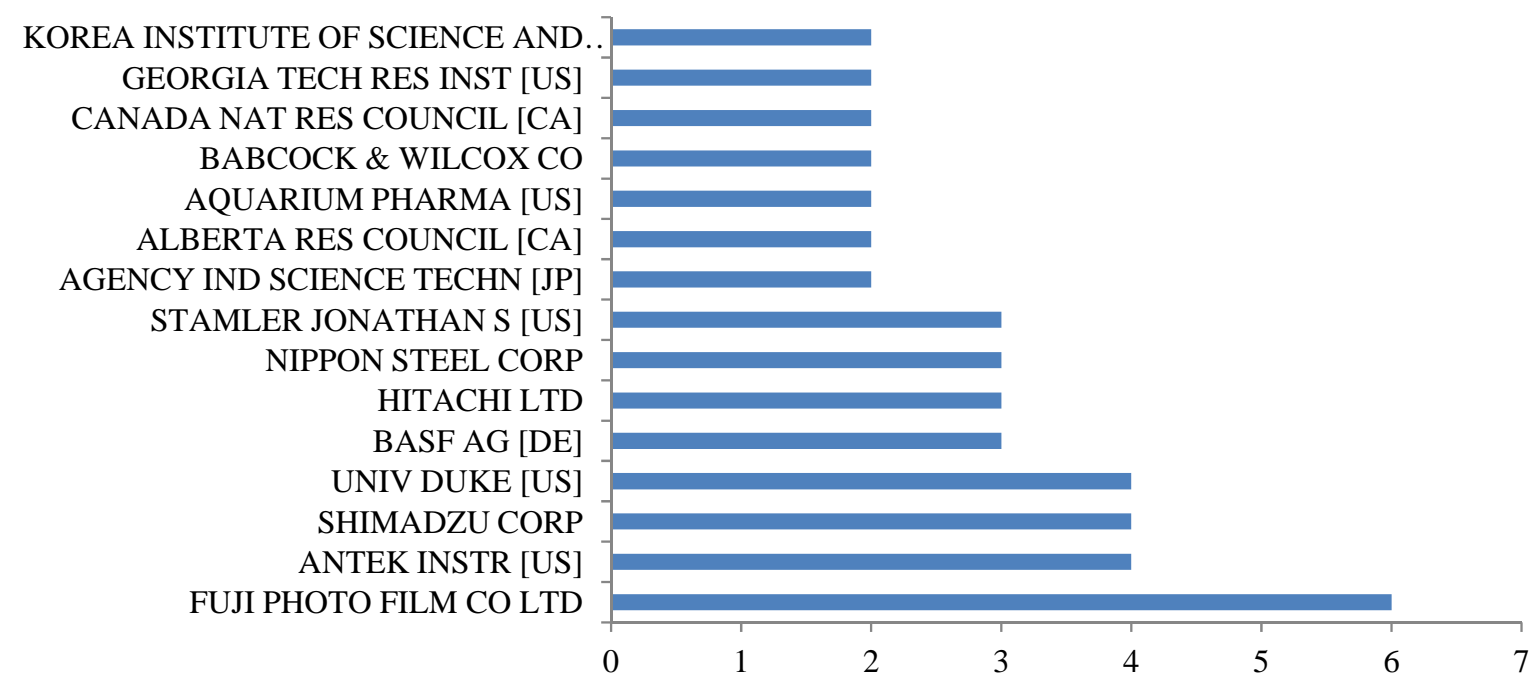

Figura 5: Número de patentes por depositantes. Fonte: Autoria própria, 2012.

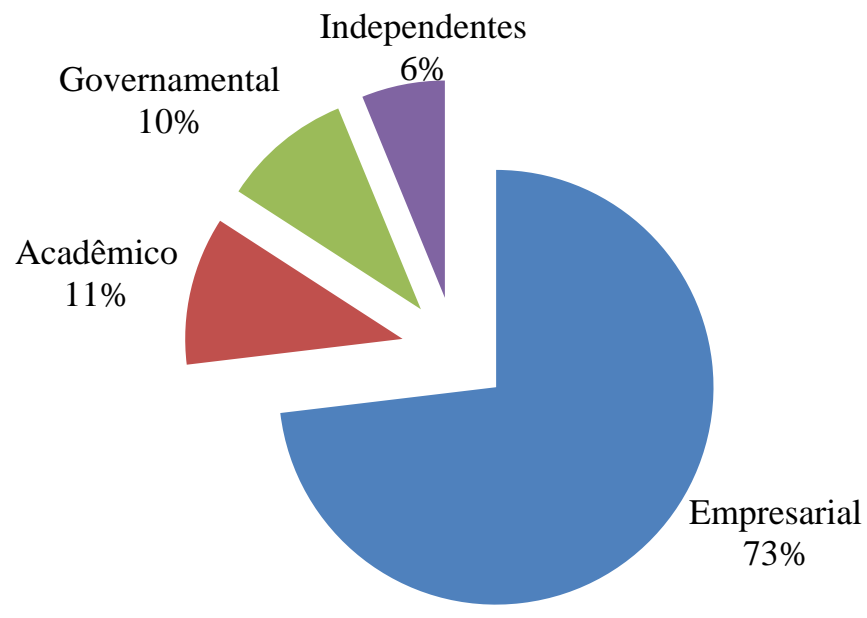

Figura 6: Distribuição das patentes depositadas no Espacenet por tipo de depositante. Fonte: Autoria própria, 2012.

Nas patentes estudadas, foi identificado também os códigos mais citados e quais as palavras-chave mais utilizadas nas patentes. Pela análise das Figuras 6 e 7, verifica-se que o código mais utilizado foi o G01N21/76, relacionado a quimioluminescência (ou bioluminescência).

Com relação às palavras chaves, verificou-se que as mais citadas foram fluorescência, oxidação e óleo. A Figura 8 apresenta esses resultados. 


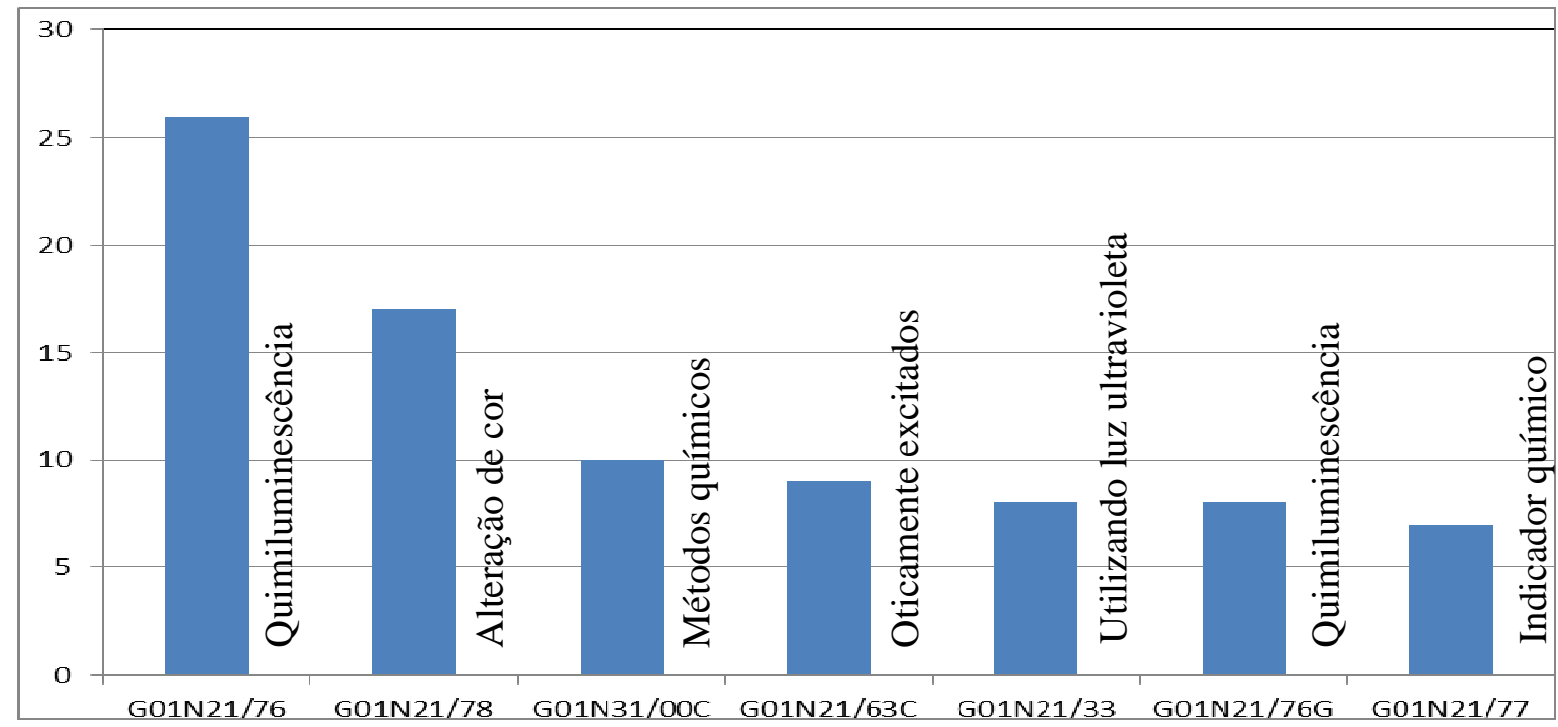

Figura 7: Número de patentes por código de maior relevância. Fonte: Autoria própria, 2012.

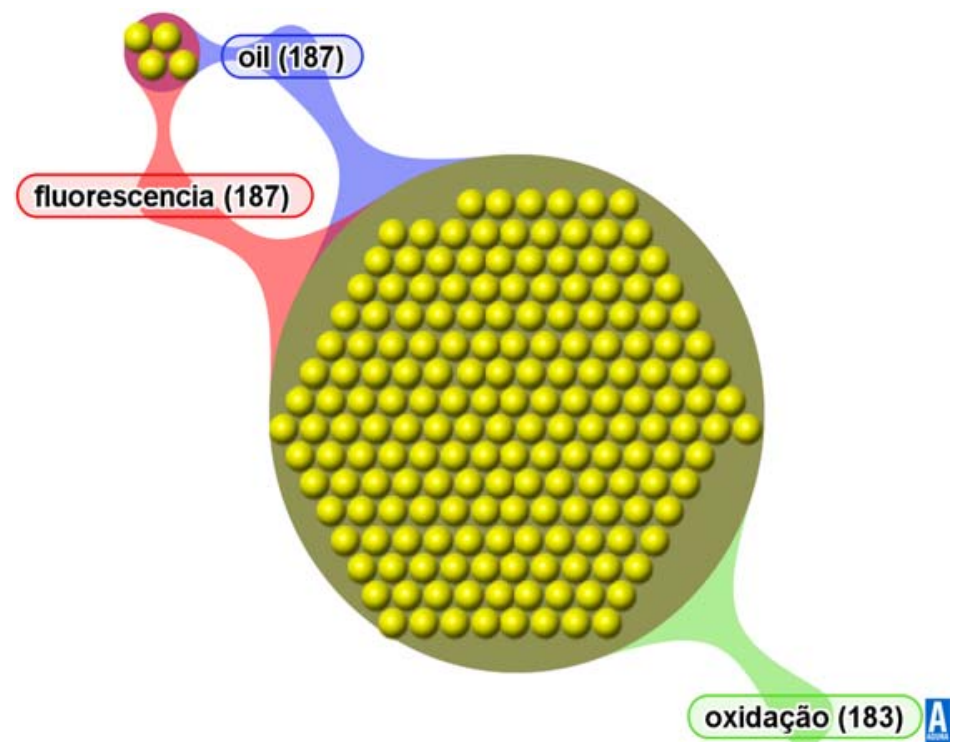

Figura 8: Relação das palavras mais citadas. Fonte: Autoria própria, 2012.

O presente trabalho também teve o objetivo de realizar a busca de anterioridade de patente de método para determinação da estabilidade oxidativa de óleos e biodiesel através do uso da 
espectrofluorimetria associada à quimiometria. Para isso, a pesquisa foi especificada por associação das palavras-chaves oxidat* and oil* com o código G01N21 sendo encontradas apenas 6 patentes. Estas foram todas lidas buscando alguma patente similar. Verificou-se que a primeira se referia a um método e um dispositivo de acompanhamento da oxidação do óleo em tempo real. O método compreende as etapas de: irradiação de luz ultravioleta em óleo a ser monitorado; medição da intensidade de emissão de fluorescência do óleo em vermelho, verde e azul de maneira a acompanhar a intensidade de fluorescência até um valor crítico que indica a necessidade de substituir o óleo por um novo.

A segunda patente se refere a um aparelho para medir a oxidação do óleo em que a parte de controle calcula um rendimento quântico de fluorescência e um coeficiente de absorção da luz do óleo e os compara com os valores de referência pré-armazenadas na parte de controle para determinar a oxidação do óleo.

A terceira patente é um processo que monitora online o grau de degradação ou oxidação de uma matéria-prima através de espectroscopia no infravermelho próximo. Um feixe de radiação no infravermelho próximo é direcionado para a superfície da amostra, por exemplo, em uma correia transportadora, para produzir radiação refletida. Distintas características espectrais são indicativas do grau de oxidação do óleo.

A quarta patente refere-se à determinação da qualidade do óleo lubrificante usado em um motor de combustão interna, compreendendo aquisição parâmetros de qualidade (oxidação, nitrificação, água, entre outros) utilizando espectrometria no inflavermelho.

A quinta patente se refere a medir a deterioração de óleos industriais causada por contaminação e oxidação através de um aparelho miniaturizado sem a necessidade de uma câmara escura através de luz com frequência constante.

A sexta patente diz respeito a três métodos. O primeiro para rápida análise, seca e não destrutiva do estado oxidativo de lipídios insaturados em alimentos integrais, gorduras e óleos. O segundo método para a previsão do tempo de armazenamento de lipídio. O terceiro método para avaliar a eficácia dos antioxidantes naturais ou sintéticos. Os três métodos dependiam da fluorescência de compostos formados a partir da reação dos voláteis resultantes da oxidação dos lipídios e epsiloncaprolactama polimerizado.

Nenhuma destas patentes sugere o uso da espectrofluorimetria combinada com calibração multivariada para predição da estabilidade oxidativa de óleos e biodieseis que foi o objeto da patente PI 011100001114 para qual se buscou a anterioridade. Esta patente se refere a um método para predição de propriedades, incluindo estabilidade oxidativa de amostras de óleos, como por exemplo, diesel, biodiesel e óleos vegetais. Mais especificamente, o método consiste na obtenção de um modelo matemático obtido entre dados de análises espectroscópicas e dados resultantes de análise de estabilidade oxidativa, obtidos por um método de referência por meio da aplicação de Análise Multivariada, tal como, Mínimos Quadrados Parciais (PLS), o qual pode ser aplicado como modelo para predição da estabilidade oxidativa de amostras desconhecidas.

Ressalta-se, no entanto, que a busca de anterioridade não verifica os documentos de patentes que estão em período de sigilo nos escritórios oficiais, que é de 18 meses. No caso da existência de algum documento similar ou igual no período de sigilo prevalece o primeiro depositante. 


\section{CONCLUSÃO}

A partir da análise dos resultados encontrados na prospecção, verificou-se que o uso de métodos óticos para determinação da oxidação de óleos é ainda pouco explorado consistindo de oportunidades para P\&D e tendo alto potencial para gerar inovação.

Existem hoje cerca de 200 patentes relacionadas a métodos óticos e oxidação, e destas, apenas 6 se referem a método, dispositivo ou aparelho para monitorar a oxidação de óleos por técnicas espectroscópicas. Das técnicas espectroscópicas apenas espectroscopia de fluorescência e espectrometria no infravermelho foram citadas nas patentes. Nenhuma das patentes sugere o uso da espectrofluorimetria combinada com calibração multivariada para predição da estabilidade oxidativa de óleos e biodieseis que foi o objeto da patente PI 011100001114 para qual se buscou a anterioridade.

A maior desvantagem dos métodos óticos em relação aos métodos clássicos existentes atualmente diz respeito ao custo do equipamento. No entanto, a necessidade de sistemas de detecção mais versáteis, rápidos e eficientes para o monitoramento da oxidação de óleos e biodiesel podem estimular o desenvolvimento, em curto espaço de tempo, de uma grande variedade de tecnologias com o uso de métodos óticos que aliados a vantagens de maior precisão e exatidão dos resultados, a possibilidade de automação e principalmente a maior rapidez analítica pode superar e muito a questão econômica.

Os métodos óticos, principalmente a espectrofluorimetria e espectrometria no IR revelam grandes potencialidades quanto a sua utilização no monitoramento da oxidação de óleos e biodiesel, sendo campos férteis de estudos e de tecnologias a serem desenvolvidas. Para suplementar estas técnicas, os biossensores podem se constituir em uma ferramenta promissora devido as suas características únicas, tais como, baixo custo relativo de construção, potencial para construção de equipamentos simples e portáteis, possibilidade de monitoramento no campo e facilidade de automação.

\section{REFERÊNCIAS}

C.M. QUINTELLA, L.S.G TEIXEIRA, M.G.A. KORN, P.R. COSTA NETO, E.A. TORRES, M.P. CASTRO, C.A.C. JESUS. Cadeia do biodiesel da bancada à indústria: uma visão geral com prospecção de tarefas e oportunidades para P\&D\&I Quim. Nova v.32. 2009.

D. BERTHIAUME, A. TREMBLAY. Study of the Rancimat test method in measuring the oxidation stability of biodiesel ester and blends. Ottawa, Ont.: Natural Resources Canada, 2006.

http://www.technopolethetford.ca/FichiersUpload/Softsystem/NRCan-OLEOTEK-

StudyoftheRancimatTestMethodinMeasuringtheOxidationStabilityofBiodieselEstersandBlends.pdf.

Accessed in February 2011.

MEIRA, M.; QUINTELlA, C. M.; COSTA NETO. P. R.; PEPE, I. M.; SILVA, H. R. G; TANAJURA, A. S. Patente tipo PI Nacional em fase de sigilo, PI 011100001114, 2010.

MEIRA, M.; QUINTELlA, C. M.; FERRER, T. M.; GONÇALVES, H. R.; KAMEI, A. G.; SANTOS, M. A.; COSTA NETO, P. R.; PEPE, I. M. Identificação de Adulteração de Biocombustível por Adição de Óleo Residual ao Diesel por Espectrofluorimetria Total 3D e Análise das Componentes Principais. Química Nova, Vol. 34, No. 4, p. 621-624, 2011. 
MEIRA, M; QUINTELLA, C. M.; TANAJURA, A. S.; SILVA, H.R.G ; FERNANDO, J. E. S.; COSTA NETO, P. R.; PEPE, I. M.; SANTOS, M. A.; NASCIMENTO, L. L. Determination of the oxidation stability of biodiesel and oils by spectrofluorimetry and multivariate calibration. Talanta, 85, p. 430-434, 2011.

MONYEM, A.; VAN GERPEN, J. H. The effect of biodiesel oxidation on engine performance and emissions. Biomass Bioenerg, v.20, p.317-325, 2001.

MONYEM, A.; VAN GERPEN, J. H.; CANAKCI, M. The effect of timing and oxidation emissions from biodiesel-fueled engines. Trans. ASAE, v.44, p.35-42, 2001.

SENDZIKIENE, E., V. MAKAREVICIENE AND P. JANULIS. Oxidation stability of biodiesel fuel produced from fatty wastes. Polish Journ.of Environ. Studies,14 (No.3), p. 335-339,2005. 